\title{
Canal équipé d'une soufflerie et d'un moyen mécanique de simulation du vent sur la plaque tournante de Grenoble
}

\author{
PAR \\ G. Chabert d'Hières \\ Ingénieur au C.N.R.S. \\ détaché à l'Institut de Mécanique de Grenoble \\ Ex \\ D. Renouard \\ Ingénieur à \\ I'Institut de Mécanique de Grenoble
}

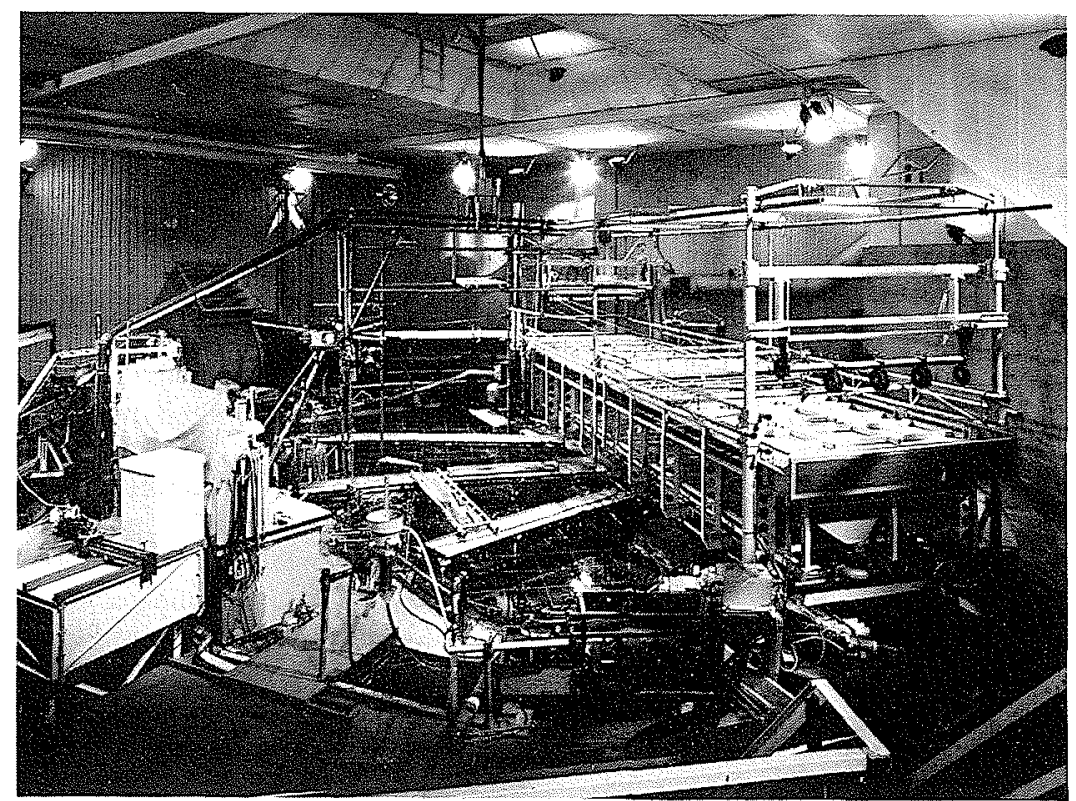

Photographie montrant l'ensemble du canal de $8 \mathrm{~m}$, équipé de la soufflerie. On distingue le portique qui supporte le mécanisme de simulation de l'effet d'entraînement dû au vent.

\section{Résumé}

L’actuel regain d'intérêt dont bénéficie l'océanographie a fait apparaitre l'impérieux besoin d'une meilleure connaissance des mouvements liés au vent, et, en particulier, des circulations et des ondes internes ou des remontées d'eau (upurelling), que celui-ci engendre. Mal- heureusement, les observations in situ sont trop rares et trop fugitives pour faire apparaître clairement les mécanismes qui interviennent, notamment lors de la formation des oscillations de la thermocline, ainsi que la structure même de ces oscillations. Par suite, le besoin d'une installation expérimentale de qualité, en milieu tournant, pour affiner ou rejeter les théories explicatives qui existent déjà, est évident. 
Sur la plaque tournante de Grenoble, plaque qui supporte actuellement le modèle réduit de la Manche, et dont on connaît les dimensions et les caractéristiques remarquables, on a donc construit un canal de $8 \mathrm{~m}$ de long, $2 \mathrm{~m}$ de large et $0,6 \mathrm{~m}$ de haut, qui permet l'étude soit d'un océan homogène, soit d'un océan stratifié de façon discrète (deux veines fluides superposées de densités légèrement différentes). Il est équipé de divers mécanismes de simulation de l'effet d'entraînement du vent :

- une soufflerie, avec laquelle on peut établir, et contrôler, dans une veine d'air surmontant le liquide, un vent de vitesse constante dans l'espace mais variable dans le temps;

- un moyen mécanique qui simule l'effet d'entraînement de la couche superficielle du liquide par le vent ;

- une circulation forcée, dans la veine supérieure du liquide, qui permet de représenter l'effet du vent intégré sur toute la hauteur de cette veine; sous réserve d'un réglage adéquat, elle permet également d'apporter quelques corrections aux circulations obtenues, dans la veine supérieure, par les deux précédents procédés.

Ce canal permettra de nombreuses études dans le domaine des ondes internes, des ondes de surface et des champs de courant, en milieu tournant. En effet, on peut l'équiper de fonds de formes diverses, de générateurs d'ondes et, le cas échéant, d'un circuit de recirculation pour la veine inférieure

Ce rapport décrit brièvement l'appareil ainsi que les divers instruments de mesures qui seront utilisés et il présente la liste, évidemment non limitative, des recherches possibles sur cette installation.

\section{Introduction}

L'existence et l'importance des ondes internes et des remontées d'eau près des côtes (upwelling) est connue depuis longtemps [cf. Stokes, 1847 (étude théorique), Watson, 1904 (premières mesures in situ), Schmidt, 1908 (première étude expérimentale)...). Cependant, en liaison avec les problèmes posés par la recherche de matières premières (pétrole, gaz, nodules métalliques) ou avec ceux soulevés par l'extension des eaux territoriales, ou de la "zone économique", notamment pour la pêche. l'actuel développement de la recherche océanographique exige, tant sur le plan théorique que sur le plan expérimental, en nature et au laboratoire, un effort accru. En particulier, l'étude des divers effets du vent (upwellings, oscillations de la thermocline, courants, ...) est d'un intérêt tout à fait primordial aussi bien pour la pêche et les conditions écologiques et donc climatiques, que pour les problemes de pollution.

Cependant. et malgré des progrès théoriques importants, plusieurs questions fondamentales restent encore sans réponse. Ceci est dû, bien sûr, à la grande complexité des processus mis en ouvre par ces phénomènes. mais également à la rareté de mesures in situ qui soient fiables et qui pourraient appuyer ou infirmer les divers schémas analytiques ou numériques en présence. On peut, à ce propos, citer cette phrase de P. M. Phillips (1966), qui nous semble bien situer le problème :

"The advances of our understanding of wind generated waves, have been consequent of the exchange between a properly argued theory on the one hand and careful and extensive observations on the other. The problems involved in internal waves are no less complex, but the difficulty of making significant measurements has, to some extent, help back a similarly fruitful exchange."

En particulier, les divers mécanismes mis en œuvre, lors de la formation puis de la propagation des ondes internes et des courants engendrés par le vent, sont encore mal définis. Ainsi Konyon (1968) a-t-il pu écrire que "les mouvements océaniques associés aux ondes internes sont observés presque partout depuis le début de ce siècle, mais leurs causes ne sont pas encore clairement connues". En effet, à ces ondes internes et à ces courants, est liée une importante turbulence, turbulence qu'il est parfois difficile de dissocier des ondes internes avec lesquelles elles peuvent coexister. A cette turbulence sont associés les problèmes de diffusion ou de mélange, dont on connaît l'importance pour les questions de pollution et de transfert d'énergie thermique. Récemment l'intérêt s'est porté sur les effets, tant à la surface libre qu'à l'interface, du passage des ondes sur le plateau continental et sur une plage.

Pour l'ensemble de ces questions, les études théoriques font clairement apparaître l'influence de la rotation terrestre, c'est-à-dire de la force de Coriolis. Or, à notre connaissance, il n'existe pas, à l'heure actuelle, d'installations expérimentales de dimensions importantes dans lesquelles on puisse simuler l'effet de cette force. En effet, les grands canaux de laboratoire, notamment ceux de Keulegan (Washington), Plate (Colorado), O'Brien (Floride) ou Shemdin (Floride) sont fixes. Nous avons donc décidé d'implanter, sur la grande plaque tournante de Grenoble, un canal que nous avons équipé d'une soufflerie, d'un moyen mécanique de simulation du vent et d'un circuit de recirculation pour la couche supérieure de liquide. Nous avons ainsi trois moyens différents pour engendrer des ondes internes. Il est prévu d'équiper, prochainement, ce canal de générateurs d'ondes et d'un second circuit de recirculation.

Nous nous proposons ici de décrire en détail ce canal et les installations de mesures que nous utiliserons, afin d'indiquer les principaux thèmes de recherche qui pourront être abordés sur cette installation.

\section{Présentation de l'installation}

Cette installation expérimentale permet, pour un liquide homogène ou stratifié en densité (de façon discrète), l'étude des courants, des ondes de surface et des ondes internes engendrées par le vent.

Elle comprend un canal dont la section d'essai a $8,0 \mathrm{~m}$ de long, $2,0 \mathrm{~m}$ de large et $0,60 \mathrm{~m}$ de profondeur, ce qui, compte tenu du "faux-fond» dont nous indiquerons plus loin la nécessité, donne une hauteur utile 

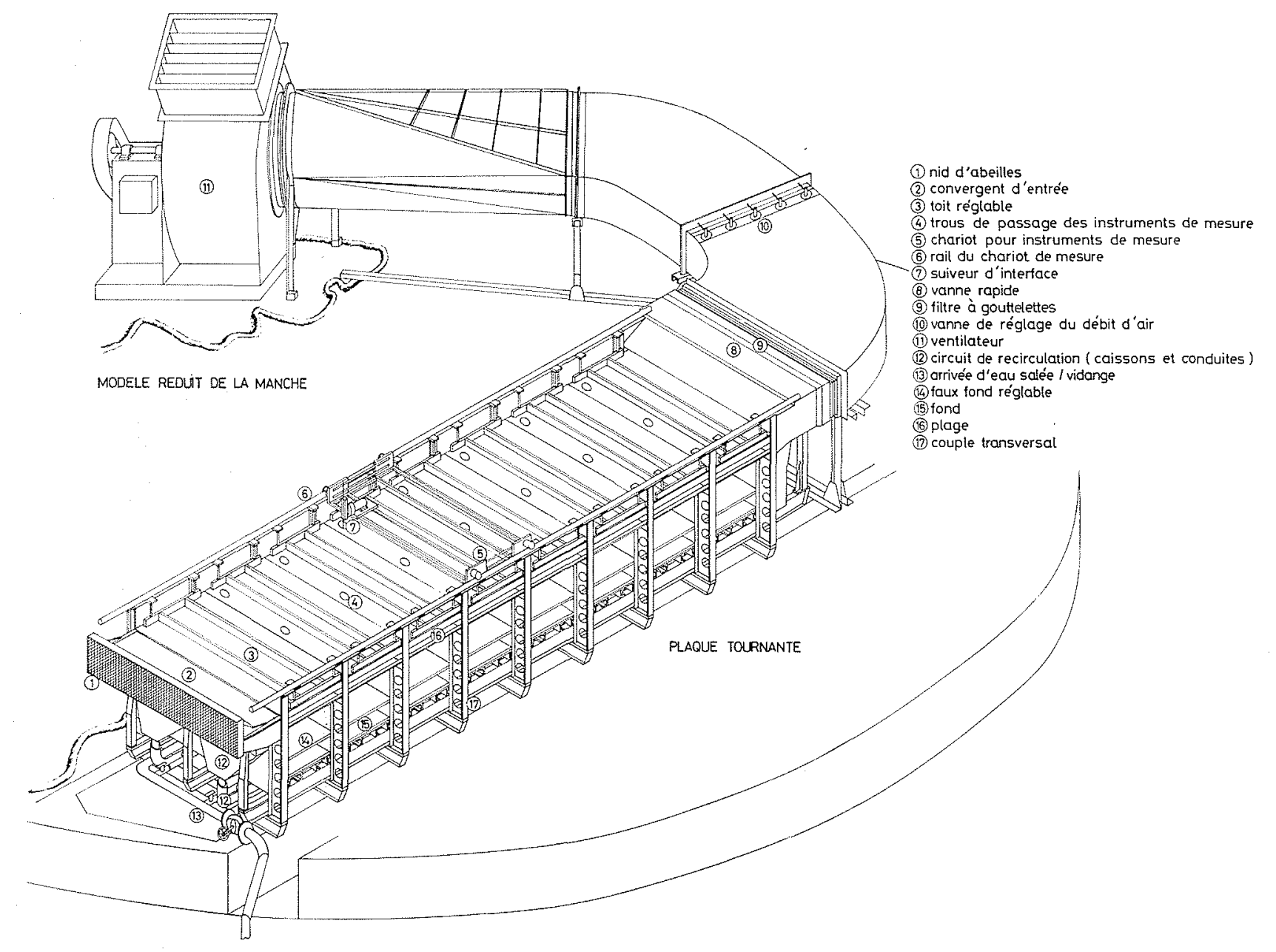

$1 /$ Vue d'ensemble de l'installation, avec la soufflerie.

de liquide variant de 0 à $0,55 \mathrm{~m}$ environ. La figure 1 est une vue d'ensemble de l'installation qui indique ses principales dimensions et permet de situer les divers éléments qui la composent.

Un ventilateur basse pression, situé à l'aval de l'installation, crée le courant d'air destiné à représenter l'action du vent sur la masse liquide de la section d'essai. L'installation est complétée par deux cuves, I'une en PVC de $1 \mathrm{~m}^{3}$, l'autre en acier émaillé de $10 \mathrm{~m}^{3}$, nécessaires à la préparation de la saumure, avec lesquelles sont obtenus les liquides de densités voulues.

\section{A. Section d'essai (cf. fig. 2)}

La partie inférieure de cette section (veine liquide) a pour parois verticales des panneaux de verre de $12 \mathrm{~mm}$ d'épaisseur et le fond est constitué par des plaques de PVC alternées avec des plaques en verre de $12 \mathrm{~mm}$ d'épaisseur. L'alternance des plaques permet de réaliser un compromis entre la charge tolérable par la plaque tournante $\left(1 \mathrm{t} / \mathrm{m}^{2}\right)$ et les dilatations thermiques admissibles pour le canal. Ces plaques de fond ont $2,0 \mathrm{~m}$ de large et $1,0 \mathrm{~m}$ de long. C'est dans les plaques en PVC que débouchent les conduits d'amenée d'eau salée, c'est également par ces conduits que peuvent être introduits quelques-uns des instruments de mesure qui exploreront l'intérieur de la masse fluide. Les plaques de verre permettront un certain nombre de mesures optiques.

Ce fond a été complété par un "faux-fond», simplement posé sur le fond proprement dit. En effet, en milieu tournant, les surfaces équipotentielles sont des paraboloïdes de révolution, et, en particulier, pour la zone occupée par notre canal sur la plaque tournante, et pour des vitesses de rotation comprises entre 0 et $4 \mathrm{tr} / \mathrm{mn}$, la cote de ces surfaces varie entre 0 et $14 \mathrm{~cm}$ environ. C'est ce qui nous a amenés à concevoir ce «fauxfond ", réglable et déformable. Ce qui permet, pour une hauteur donnée, d'ajuster la forme du "faux-fond" à celle du paraboloide équipotentiel correspondant à la vitesse de rotation choisie pour l'expérience.

La partie supérieure de cette section d'essai (veine d'air) a $0,20 \mathrm{~m}$ de haut et $2,40 \mathrm{~m}$ de large. La veine d'air est plus large que la veine liquide pour assurer, au-dessus de celle-ci, une vitesse d'air aussi constante que possible le long d'une ligne transversale, par élimination des effets de bords. Les panneaux verticaux sont, comme pour la veine liquide, en verre, de $12 \mathrm{~mm}$ d'épaisseur et $0,30 \mathrm{~m}$ de haut. Le toit est constitué par 
(1) parois VERTicales a/veine élargie panneaux de verre 100.30$\}$ colle sur $\mathrm{b} /$ canal ponneaux de verre 100.60 , armature ocie

(2) FOND

FOND

d/ploque de verre collé sur

(3) ARMATURE i couple transwersal

(4) Faux AUX FOND déformable et réglable en oltitude
h/plaque perforée recouverte d'une plaque pleine amovible i/pieds réglables

(5) COTE prévie pour la surface libre du llquide

(6) TOIT REGLABIE

$k$ /rous de possage des instruments de mesures CONVERGENT D'ENTRÉE I nids d'abeilles
$\mathrm{m} /$ filtres tergal

(8) CIRCUIT DE RECIRCULATION n/caissons de réportition amont et avol $0 /$ grille d'entre
$\rho /$ débitmètres

(9) CIRCUIT D'ALIMENTATION q/alimentation de 4 fonds s/vidange

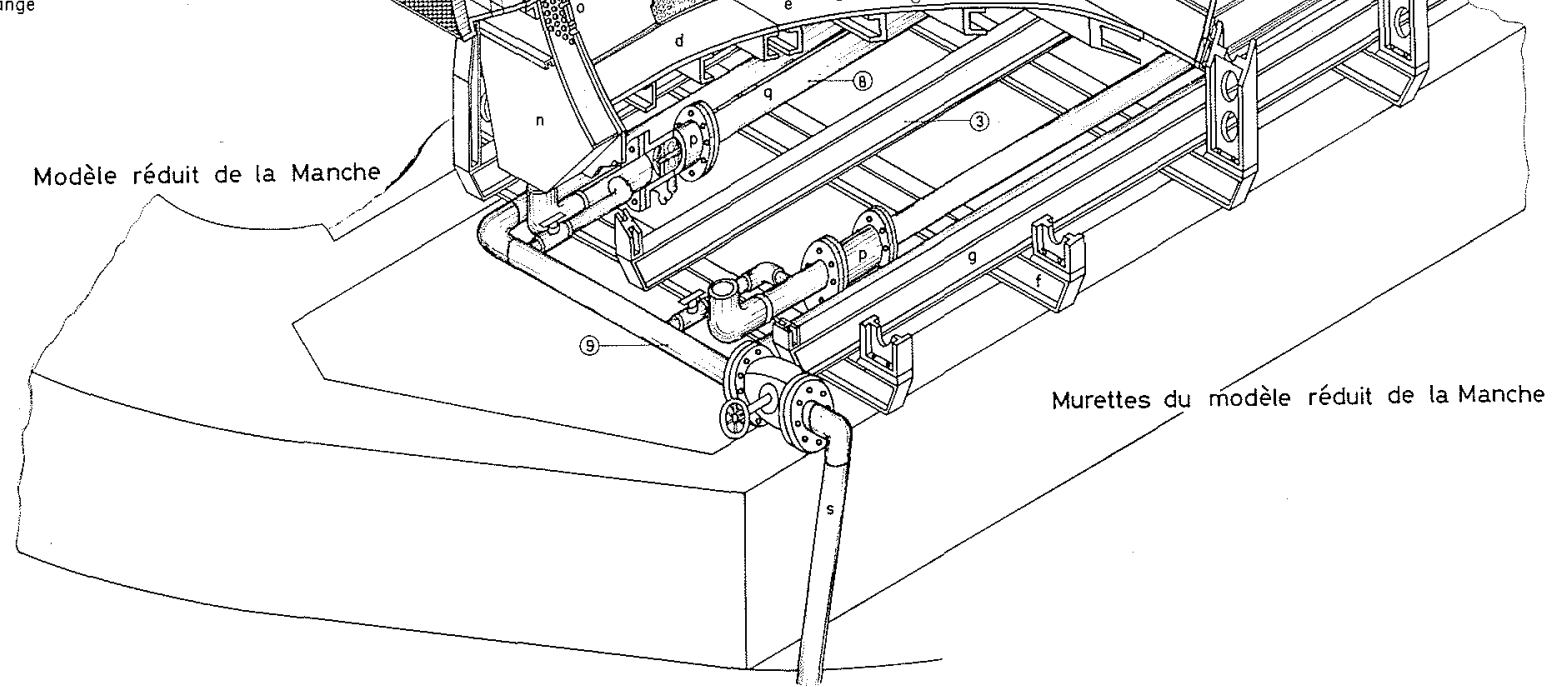

2/ Détail de la section amont, l'installation étant équipée de la soufflerie.

des plaques de PVC, il est déformable et réglable en hauteur. ce qui permet d'établir une légère compensation de la perte de charge par un réglage judicieux de la section d’air. Ce toit est aménagé pour permettre le passage de divers instruments de mesure.

\section{B. Circuit d'air (cf. fig. 2)}

Le circuit d'air, ouvert, est composé, à l'amont, d'un convergent, de filtres et de nids d'abeilles qui régularisent l'écoulement, puis d'une plage de raccordement air-eau et de la veine d'essai décrite ci-dessus. Immédiatement après cette veine d'essai, nous avons placé une vanne à ouverture (fermeture) rapide qui permet de réaliser un vent impulsionnel dans le temps. Elle est suivie d'un filtre, qui joue le rôle de "piège à goutteletles d'eau ". puis d'un répartiteur de débit. Une gaine de raccordement entre le canal et le ventilateur complète ce circuit. Cette gaine est indépendante à la fois du canal et du ventilateur afin que les vibrations de celui-ci ne se transmetlent pas au canal. Le ventilateur permet de réaliser. dans la veine d"essai, une vitesse d"air, réglable. comprise entre () el $20 \mathrm{~m} / \mathrm{s}$. Une vanne de réglage du débit est placée en aval du ventilateur.

\section{Simulation du vent par un moyen mécanique (cf. fig. 3)}

Obtenir un vent impulsionnel d'intensité et de direction constantes, soit une tension constante sur une surface de $16 \mathrm{~m}^{2}$, est très délicat. Aussi avons-nous eu l'idée de simuler l'effet d'entraînement du vent par un moyen mécanique, d'un emploi plus souple et d'une plus grande fiabilité. Pour justifier cette substitution, nous rappelons que, pour résoudre les équations classiques de l'hydrodynamique marine, on intègre les vitesses suivant une verticale et on ne s'intéresse plus alors qu'à des débits par unité de largeur de l'océan ou du canal. Si donc on est en similitude, quant aux débits, entre la nature et le canal, quelle que soit la loi de vitesse suivant une verticale, loi d'ailleurs très mal connue dans la nature, on sera en similitude quant aux dénivellations de l'interface.

La limite de ce postulat est l'influence réelle de cette loi de vitesse suivant une verticale, influence que la théorie masque complètement et que seule l'expérience révèlera.

On a procédé, sur un petit canal annexe, à de nombreux essais préliminaires afin d'établir un choix parmi les divers moyens mécaniques imaginés pour assurer 


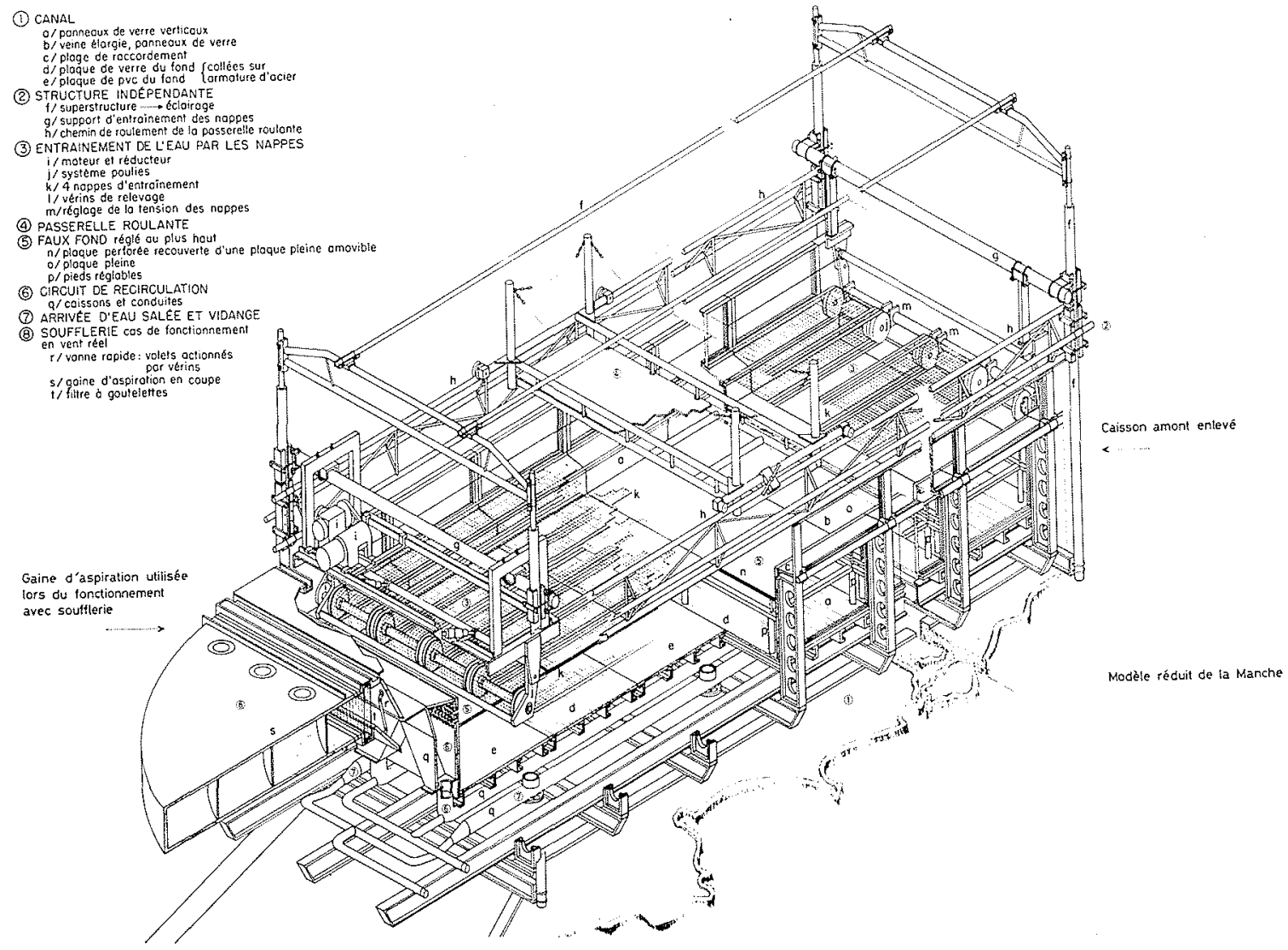

3/ Détail de l'extrémité aval, l'installation étant équipée du mécanisme de simulation de l'effet d'entrainement du vent.

cette fonction, et de vérifier le bon fonctionnement du mécanisme retenu. Finalement, nous avons opté pour un grillage (à maille carrée de $2 \mathrm{~cm}$ de côté, tendu par des cordeaux en acier) que l'on déplace à la surface de l'eau suivant une loi de mouvement que l'on peut choisir à volonté.

Sur le grand canal, on a installé quatre nappes parallèles d'un tel grillage, montées en tapis sans fin sur des rouleaux entraînés par un moteur à vitesse constante, mais réglable. Le grillage peut être appliqué (ou relevé) rapidement de façon à traduire effectivement, en tout point de la surface, le caractère impulsionnel du vent dont nous désirons étudier les effets.

\section{Circuit de recirculation}

Nombreuses seront les expériences dans lesquelles on devra établir et contrôler les circulations dans la veine supérieure du liquide. Par exemple, si l'on ne désire pas limiter l'étude au cas des lacs ou des océans rectangulaires, mais l'étendre au cas d'un canal de longueur «infinie» (au moins pour la couche supérieure dans le cas d'un océan stratifié de façon discrète), ou si l'on veut étudier les phénomènes liés à un courant (dans le fluide supérieur seul), ou encore si l'on souhaite connaître les effets d'une interaction entre le vent et un courant, alors il est nécessaire de prévoir un circuit de recirculation extérieur au canal.

Ce circuit comprend les deux caissons d'extrémité du canal et deux conduits, parallèles, qui relient ces caissons, et dans lesquels le mouvement du fluide peut être mesuré et contrôlé à l'aide de débitmètres et d'injecteurs.

\section{E. Alimentation en eau douce et eau salée}

Le schéma d'alimentation en eau douce et eau salée (fig. 4) montre la relative complexité de cette double installation qui doit assurer le remplissage du canal alors que la plaque est en rotation à la vitesse choisie pour l'expérience. En effet, une variation importante de la vitesse de rotation de la plaque, alors que le canal est déjà rempli ou en cours de remplissage, entraîne un mélange intolérable.

L'eau douce est celle de la ville que l'on dégaze et réchauffe pour l'amener à la température ambiante. Le sel est d'abord dissous dans une petite cuve de $1 \mathrm{~m}^{3}$. puis cette saumure, concentrée à saturation, est diluée 

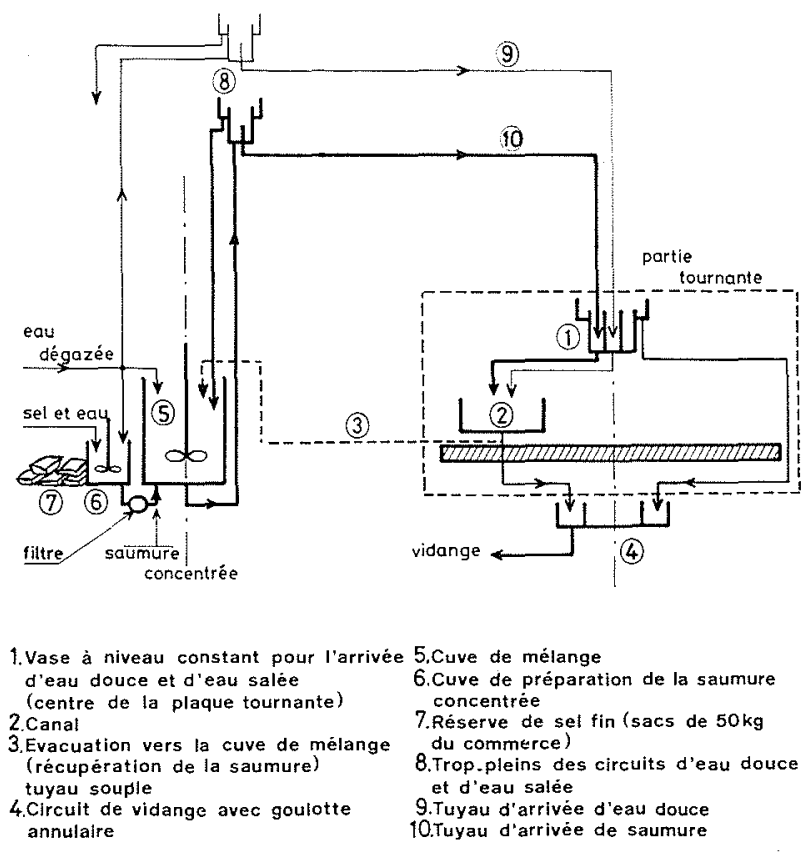

$4 /$ Schéma de principe de l'allmentation du canal de $8 \mathrm{~m}$ en eau douce el eau salée.

dans une cuve de $10 \mathrm{~m}^{3}$ en acier émaillé, pour obtenir la concentration désirée. Cette dernière sert également au stockage de la solution. Deux puissants agitateurs assurent l'homogénéité des solutions dans chacune des cuves. L'opération de remplissage du canal dure environ trois heures.

\section{Instruments de mesure}

Les expériences que nous avons réalisées sur le petit canal de $6 \mathrm{~m}$, ainsi que les diverses publications que nous avons consultées, nous amènent à prévoir :

1. Pour les hauteurs: dans un premier temps, un limnimètre à pointe manuelle Neyrpic et, plus tard, un appareil à ultra-sons plus adapté.

2. Pour les vitesses et pressions d'air : des tubes de Pitot dont les pressions sont lues et enregistrées à l'aide de micromanomètres Disa.

3. Pour les mesures de turbulence dans l'air, un anémomètre à fil chaud, associé à un appareil d'enregistrement du signal Intertechnique (analyseur multicanaux). Le même système pourra servir pour les mesures de turbulence dans l'une ou l'autre des couches liquides.

4. Pour les mesures de vitesses dans l'eau, nous emploierons un micro-moulinet (courantomètre fabriqué par le Delft Hydraulic-Laboratory) en parallèle avec des traceurs colorés et des micro-bulles d'hydrogène, que l'on photographiera à des instants successifs. Cette dernière méthode est efficace mais d'un emploi un peu lourd. Dans un proche avenir, la mesure de deux composantes des vitesses et de leurs fluctuations sera effec- tuée par un laser que l'on met au point au laboratoire. En ce qui concerne la vitesse de la couche superficielle, nous pensons que seule, la méthode de chronophotographie de petits flotteurs convenablement lestés, sera significative.

Pour terminer, signalons que, pour connaître la vitesse moyenne dans les conduites de recirculation, chacune d'elles sera bientôt équipée d'un débitmètre à hélice Faure-Herman.

5. Pour les variations de pression au fond et à l'intérieur de la masse fluide, on utilisera des capteurs ultrasensibles Techmation.

6. Pour les dénivellations de la surface libre, on utilisera des limnigraphes à pointe vibrante Neyrpic: à plus long terme, nous pensons utiliser un laser pour mesurer la pente des petites vagues à la surface libre et à l'interface.

7. Pour les dénivellations de l'interface, nous utiliserons les suiveurs d'interface mis au point par le Laboratoire et déjà en service sur des manipulations de haute précision et qui seront adaptés aux grands débattements que nous rencontrerons dans nos études. Ces appareils, qui sont des conductimètres asservis à suivre une couche de conductivité donnée, permettent des enregistrements analogiques immédiatement exploitables, et des enregistrements digitaux qui sont ensuite dépouillés sur l'ordinateur I.B.M. 1130 dont dispose l'I.M.G. ou sur l'ordinateur 360/70 I.B.M. du Centre Inter-Universitaire de Calcul.

La sérialisation et la mise sur bande perforée des données recueillies par ces divers instruments sont effectuées par une chaîne d'acquisition de données $G$. Klar.

Dans l'avenir, il est prévu de contrôler en continu la densité des deux couches lors du remplissage ainsi que la température en de nombreux points du canal, dans l'une ou l'autre couche.

Les nappes de grillages disposent de moteur à vitesse régulée, des tachymètres permettront de contrôler voire d'ajuster celle-ci tout au long des expériences.

Un chariot à deux mouvements croisés permet d'amener les instruments de mesures au-dessus des points de mesure. Ceux-ci sont matérialisés, dans le cas du système mécanique de simulation, par des bandes de quelques centimètres de large entre les nappes constituant ce tapis, et dans le cas de la soufflerie, par un réseau de trous dans le toit.

Un chariot, supporté par des rails et des portiques, absolument indépendant du canal lui-même, permet au manipulateur de circuler au-dessus du canal et de placer et surveiller les instruments de mesure.

\section{4. Études possibles sur le canal}

Le canal, installé sur la plaque tournante, permet l'étude d'un grand nombre de phénomènes, en milieu tournant ou non. 
On pourra, par exemple, et, bien sûr, cette liste n'est nullement limitative :

- étendre, en milieu tournant ou non, les résultats expérimentaux ou théoriques de Plate, O'Brien, Welander ou Keulegan (étude des ondes de surface, des pentes de la surface libre et de l'interface, des distributions de vitesses, de la loi de diffusion de l'eau salée dans l'eau douce, de la stabilité de l'interface, de la couche d'Ekman, ...);

- étudier des amphidromies de Kelvin et de Poincaré;

- étendre les études des ondes dańs une cuve parallélépipédique, en présence de deux couches fluides de densités légèrement différentes, et étudier les phénomènes non linéaires qui sont liés à ces ondes, ceci en prolongement des expériences et des études théoriques actuellement en cours dans une petite cuve de $2 \times 0,5 \times 0,4 \mathrm{~m}$;

- étudier les ondes de berge ou de plateau et le passage d'une onde sur le plateau continental (en milieu stratifié ou non); ceci au prix de l'adaptation, sur le canal, de batteurs ou d'obstacles appropriés, cette adaptation ne posant d'ailleurs aucun problème particulier de par la conception même du canal;

- étudier systématiquement la reproduction, sur modèle réduit, de l'effet du vent sur des phénomènes océanographiques à grande échelle, et des problèmes de similitude qui y sont liés (cf. grands courants océaniques), mesurer la répartition verticale des vitesses horizontales en milieu tournant, stratifié ou non, et reproduire les mouvements tourbillonnaires à moyenne échelle;

- étudier les efforts sur les structures (tube de remontée de pétrole brut, sous-marins, ...) des champs de courants singuliers liés aux gradients de densité;

- étudier les problèmes de mélange ou de diffusion pour les rejets de polluant en présence d'une interface et de vent;

- étudier les ondes internes engendrées par le vent.

De telles études, fort coûteuses dans la nature, ne peuvent être faites de façon systématique et détaillée, tout en restant réalistes, que sur un modèle physique de grandes dimensions. C'est pourquoi nous pensons que, dans ces domaines de recherche qui sont d'actualité, cette installation, confirmant des études analytiques ou numériques, saura susciter l'intérêt de nombreux théoriciens ou utilisateurs.

\section{Remerciements.}

Ce canal a pu être réalisé grâce à l'aide du C.N.R.S. dans le cadre d'une "action spécifique ". Son équipement se poursuit dans le cadre de l'Action thématique programmée « océanographie physique ».
Les études ont été exécutées au bureau d'études de l'I.M.G. La construction a été faite en partie à l'atelier de I'I.M.G. et en partie par les ateliers A.G.C.M. Le montage a été réalisé par l'un de nous et $M$. Bernard Rey.

\section{Quelques chiffres}

\section{A. La plaque tournante de Grenoble}

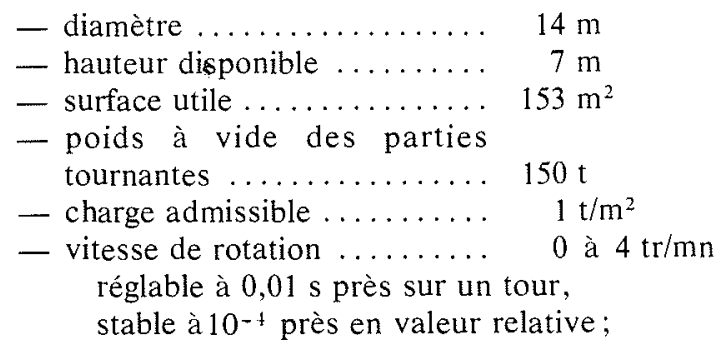

- verticalité de l'axe de rotation : les écarts à la verticale sont inférieurs à $7 \cdot 10^{-7}$ radian : tout point de la structure tournante reste, au cours des mouvements, entre deux plan horizontaux de $0,02 \mathrm{~mm}$.

\section{B. Le grand canal}

- longueur ...............

- largeur de la veine liquide... $(2,0000 \pm 0,0005) \mathrm{m}$

- largeur de la veine d'air ... $(2,4000 \pm 0,0005) \mathrm{m}$

- hauteur de la veine liquide .. $\quad(0,6000 \pm 0,0005) \mathrm{m}$

— poids du canal (à vide) ..... $\sim 6 \mathrm{t}$

- poids de la gaine de raccordement $\ldots \ldots \ldots \ldots \ldots \ldots \ldots \sim 1,3 \mathrm{t}$

- poids du ventilateur........ $\sim 1,2 \mathrm{t}$

- vitesse de l'air ........... $0<V \leqslant 20 \mathrm{~m} / \mathrm{s}$

- vitesse du tapis roulant..... $4<V \leqslant 12 \mathrm{~cm} / \mathrm{s}$

\section{Bibliographie}

Dalverny (J.) et Fontanet (F.). - La Houille Blanche, 1959, 5. Chabert d'Hières (G.). - La Houille Blanche, 1962, 2. Chabert D.Hières (G.) et Suberville (J. L.). - La Houille Blanche, 1974, 7/8.

Plare (E.). - La Houille Blanche, 1965, 6.

KEULEGAN (G. H.). - N.B.S., 1960, 6638.

Keulegan (G. H.) et Carpenter (L. H.). - N.B.S., 1961, 7319.

O'Brifn (J. J.). - J. Res. Hyd., 1971, 9, 2.

Sheppard (D. M.), Shemdin (O. H.) et Wang (Y. H.). C.O.E.L., 1973, 19. 